Document downloaded from:

http://hdl.handle.net/10251/35856

This paper must be cited as:

Villanueva Micó, RJ.; Cortés López, JC.; Santonja, F.; Morales, A. (2012). Analysing the effect of public health campaigns on reducing excess weight: A modelling approach for the Spanish Autonomous Region of the Community of Valencia. Evaluation and Program Planning. 35(1):34-39. doi:10.1016/j.evalprogplan.2011.06.004.

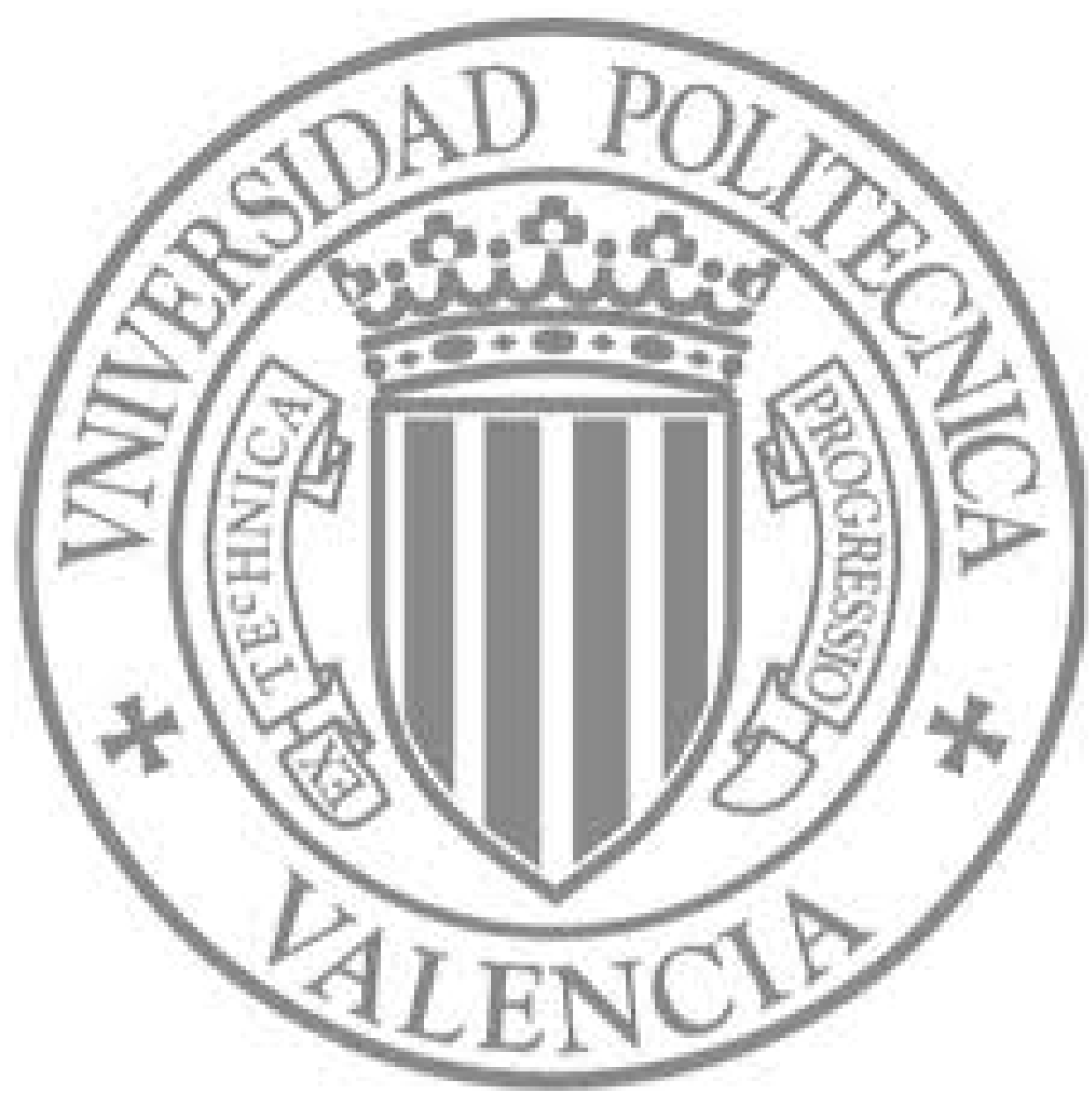

The final publication is available at

http://dx.doi.org/10.1016/j.evalprogplan.2011.06.004

Copyright Elsevier 


\section{Working paper}

El siguiente trabajo "Analysing the effect of health communication campaigns on reducing excess weight: $A$ modelling approach for the Spanish region of Valencia" ha sido remitido para su posible publicación a la revista "Evaluation and Program Planning" y trata de predecir la prevalencia de la obesidad en la población de la Comunidad Valenciana en los próximos años y estudiar el efecto de unas posibles campañas de salud pública sobre la reducción del número de personas con exceso de peso. 


\title{
Analysing the effect of health communication campaigns on reducing excess weight: A modelling approach for the Spanish region of Valencia.
}

\author{
F.-J. Santonja ${ }^{\mathrm{a}, 1}$, A. Morales ${ }^{\mathrm{b}}$, R.-J. Villanueva ${ }^{\mathrm{c}}$, J.-C. Cortés $^{\mathrm{c}}$ \\ ${ }^{a}$ Departamento de Estadística e Investigación Operativa. Universidad de Valencia, Spain. \\ ${ }^{b}$ Servicio de Endocrinología y Nutrición. Hospital de Manises (Valencia), Spain. \\ ${ }^{c}$ Instituto de Matemática Multidisciplinar. Universidad Politécnica de Valencia, Spain.
}

\begin{abstract}
Excess Weight is becoming a serious health concern that is increasing in developed and developing countries. Public health authorities are worried about it and develop health communication campaigns to inform the population the health problems that may be derived from excess weight and change their nutritional behaviour. Recent studies determine that the average health campaign affects the intervention community by about $5 \%$. The aim of this paper is to quantify the effect of different health campaigns on the population over the next few years changing the dietary behaviour and, consequently, reducing the weight of a significant number of people. This study is based on recent works that consider excess weight as a consequence of the transmission of unhealthy lifestyles from one individual to another. Under this point of view a model is presented and policies based on communication health campaigns addressed to avoid people gaining weight (prevention) and policies addressed to excess weight individuals for reducing their weight (treatment) are simulated in order to evaluate their effectiveness. The study concludes that preventive strategies are more effective than the treatment ones.
\end{abstract}

Key words:

Excess weight, Modelling, Preventive policies, Treatment policies, Health communication campaigns, Quantification, Simulations

\section{Introduction}

Excess weight population are growing at a worrying rate in developed and developing countries [1]. In the region of Valencia (Spain) it is becoming a se-

\footnotetext{
${ }^{1}$ Corresponding author.

Department of Statistics and Operations Research

University of Valencia, Valencia 46100, Spain.

Tel.: +34963544313. Fax: +34963543238

E-mail address: francisco.santonja@uv.es
} 
rious health concern not only from the individual health point of view but also from the public socioeconomic one. We note that the percentage of Valencian individuals who are obese is increasing $(11.6 \%$ in year 2000 and $13.48 \%$ in year 2005) $[2,3]$. The SEEDO (Spanish Society for the Study of the Obesity) considers the study of the obesity of highest priority evaluating its magnitude and proposing effective strategies in order to invert this trend in the next few years [4].

In this article, we assume this challenge studying the evolution of the proportion of adult valencian inhabitants (24-65 years old) with excess weight over the next few years and examine the impact of some obesity control strategies in reducing obese subpopulation. We analyse the following two proposals considered by the Valencian Department of Health in the Valencian Health Plan (2005-2009) [5]:

- To promote healthy lifestyles (healthy nutritional habits and physical activity).

- To define specific strategies to reduce overweight and obese subpopulations.

Public health authorities propose health communication campaigns following the above main lines. Recent studies $[19,20,21]$ show that the average health campaign affects the intervention community by about 5 percentage points, and nutrition campaigns for fruit and vegetable consumption, fat intake, and breastfeeding, have been slightly more successful on average than for other health topics. Moreover, in [21] the author concludes that nutrition campaigns that pay attention to the specific behavioural goals of the intervention, target populations, communication activities and channels, message content and presentation, and techniques for feedback and evaluation should be able to change nutrition behaviour.

To address this problem, we assume that excess weight (obesity and overweight) is a socially transmitted epidemic disease $[6,7]$. We treat excess weight as a disease that spreads through social peer pressure or social contact. These social contacts have an influence on the probability of transmission of sedentary lifestyles and unhealthy nutritional habits. The main idea behind this approach is that unhealthy lifestyles (unhealthy nutritional habits and sedentary lifestyles) may spread from one person to another. These facts lead us to propose an epidemiological-type mathematical model to study the evolution (transmission dynamics) of the excess weight prevalence in the population (24-65 years old) of the region of Valencia where several health strategies to be implemented in communication campaigns by the Valencian Department of Health will be analysed.

There are some papers where the authors study the dynamic evolution of the population with excess weight. For instance, in [8] a model is presented and a long-term study has been carried out. In $[9,10]$ two models are proposed, one of them age-structured, and applied to available data in the Spanish region of Valencia to predict the evolution of the excess weight children over the next 
few years. A similar study has been done for adult Valencian population in [11]. However, to our knowledge, simulations of the effect of policy strategies on dynamic models only appears in [12] for cocaine abuse.

The paper is organized as follows. The model used to simulate the effects of the health campaigns is presented and its parameters estimated in the Methods Section. In the Results Section, the evolution of excess weight population in the region of Valencia (Spain) over the next few years is shown under two points of view, and several public health strategies to reduce the excess weight population based on communication campaigns are defined. In the Discussion Section, we analyse the effect on the population of these public health strategies and compare them. The Conclusion Section will finish the paper.

\section{Methods}

\subsection{Building the model}

Valencian adult individuals (24-65 years old) are divided into three subpopulations depending on their Body Mass Index $\left(B M I=\right.$ Weight $/$ Height $\left.^{2}\right)$ : $N$, individuals with normal weight $(B M I<25) ; S$, people with overweight $(25 \leq B M I<30)$ and $O$, obese individuals $(B M I \geq 30)$.

We build the epidemiological mathematical model considering the following assumptions:

1. We assume population homogeneous mixing, that is, each individual can contact with any other individual [13].

2. Also, we consider that sedentary lifestyles and unhealthy nutritional habits increase the individual weight of the adults in the region of Valencia. Hence, the transition to overweight supopulation $(S)$ or obese subpopulation $(O)$ are determined by the transmission of unhealthy lifestyles (sedentary and unhealthy nutritional habits). The transitions described can be modelled as follows:

- An individual in $N$ transits to $S$ because people in $S$ or $O$ transmit unhealthy lifestyles by social contact at rate $\beta$. Therefore, this is a nonlinear term modelled by $\beta N(S+O)$.

- An individual in $S$ transits to $O$ at rate $\gamma$ proportionally to the size of $S$ if he/she maintains his/her unhealthy lifestyle. Hence, this is a linear term modelled by $\gamma S$.

- An individual in $S$ or $O$ transits to $N$ or $S$ respectively, when he/she decides to give up his/her unhealthy lifestyle and starts with the practice of physical activity and puts on diet. An individual in $S$ transits to $N$ at rate $\rho=\rho_{1} * \rho_{2} * p$ proportionally to the size of $S$ (modelled by $\left.\left(\rho_{1} * \rho_{2} * p\right) S\right)$ and individuals in $O$ transit to $S$ at rate $\epsilon=\epsilon_{1} * \epsilon_{2} * p$ proportionally to the size of $O$ (modelled by $\left.\left(\epsilon_{1} * \epsilon_{2} * p\right) O\right)$.

3 . We consider that the new recruited 24-year-old individuals become members of $N, S$ or $O$ proportionally to the size of 23 years old normal weight 
$\left(N_{0}\right)$, overweight $\left(S_{0}\right)$ or obese subpopulations $\left(O_{0}\right)$, respectively. They are three linear terms modelled by $\mu N_{0}, \mu S_{0}$ and $\mu O_{0}$ respectively.

4. Individuals leave of the system when they are 65 years old. This fact is proportional to the size of the subpopulations. We model it using $\mu N, \mu S$ and $\mu O$ respectively.

Then, the transitions between these different subpopulations are described by the following system of differential equations ( $t$, time in weeks),

$$
\begin{aligned}
N^{\prime}(t)= & \mu N_{0}-\mu N(t)-\beta N(t)[S(t)+O(t)]+\left(\rho_{1} * \rho_{2} * p\right) S(t) \\
S^{\prime}(t)= & \mu S_{0}+\beta N(t)[S(t)+O(t)]- \\
& -\left[\mu+\gamma+\left(\rho_{1} * \rho_{2} * p\right)\right] S(t)+\left(\epsilon_{1} * \epsilon_{2} * p\right) O(t) \\
O^{\prime}(t)= & \mu O_{0}+\gamma S(t)-\left(\mu+\epsilon_{1} * \epsilon_{2} * p\right) O(t)
\end{aligned}
$$

where the parameters of the model are:

- $\mu$, average stay time in the system of 24-65 years old adults.

- $\beta$, transmission rate to adopt unhealthy lifestyles because of social pressure.

- $\gamma$, the rate at which overweight 24-65 years old adults become obese because of unhealthy lifestyles.

- $\rho=\rho_{1} * \rho_{2} * p$ is the rate at which overweight individuals become normal weight,

- $\rho_{1}$ measures the proportion of overweight population who do physical activity in order to reduce weight.

- $\rho_{2}$ measures the proportion of overweight population who put on diet to reduce weight.

- $p$ measures the time an individual needs to return to overweight subpopulation (from obese subpopulation) or normal weight subpopulation (from overweight subpopulation) by diet and physical activity. It is assumed to be the same.

- $\epsilon=\epsilon_{1} * \epsilon_{2} * p$ is the rate at which overweight individuals become normal weight.

- $\epsilon_{1}$ measures the proportion of obese population who do physical activity in order to reduce weight.

$-\epsilon_{2}$ measures the proportion of obese population who put on diet to reduce weight.

- $p$ has been already described above.

The model diagram is depicted in Figure 1. 


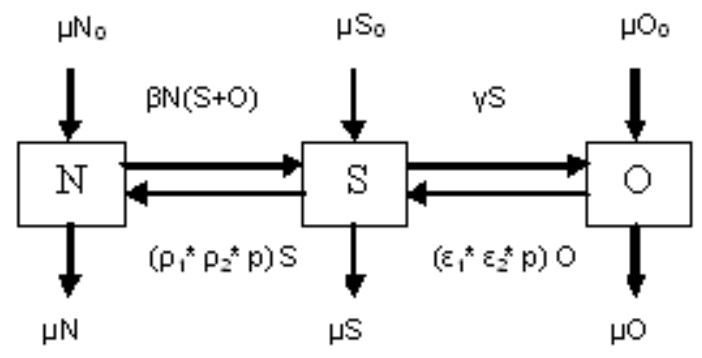

Figure 1: Flow diagram of the mathematical model for the dynamics of excess weight (overweight and obesity) prevalence in the population of the region of Valencia (Spain). The boxes represent the subpopulations and the arrows represent the transitions between the subpopulations. Arrows are labelled by their corresponding model parameters.

\subsection{Parameters estimation}

We estimate all parameters of the model except $\beta$ and $\gamma$ using the following sources:

- Health Survey for region of Valencia, year 2000 [2].

- Health Survey for region of Valencia, year 2005 [3].

- Two tecnical reports published by M.A. Rubio et al. in 2007 and W.C. Miller, D.M. Koceja \& E.J. Hamilton in 1997 [14, 15]. In these reports appear some details about strategies to reduce BMI.

Then, the parameters of the model are estimated as follows:

- $\mu=1 / 2184=0.0004578$ weeks $^{-1}$. We consider the mean time spent by an individual in the system, 42 years, i.e., 2,184 weeks.

- $\epsilon_{1}$. This value is estimated from Health Survey of the region of Valencia (year 2000) taking into account that the percentage of obese subpopulation who practice physical activities $\left(0.4 \% ; \epsilon_{1}=0.004\right)$,

- $\epsilon_{2}$. This value is estimated from Health Survey of the region of Valencia (year 2000) taking into account the percentage of obese subpopulation who control their nutritional habits $\left(2.4 \% ; \epsilon_{2}=0.024\right)$,

- $p=1 / 7$ weeks $^{-1}$. An average overweight or obese individual needs to reduce his/her weight a mean of $7 \mathrm{Kg}$ to transit to normal weight or overweight, respectively $[2,3]$. Also, a typical program of 24 -week diet plus exercise, produces a weight loss of about $1 \mathrm{Kg}$ per week [14, 15]. 
- $\rho_{1}$. This value is estimated from Health Survey of the region of Valencia (year 2000) taking into account the percentage of overweight subpopulation who practice physical activities $\left(2 \% ; \rho_{1}=0.02\right)$,

- $\rho_{2}$. This value is estimated from Health Survey of the region of Valencia (year 2000) taking into account the percentage of overweight subpopulation who control their nutritional habits $\left(4.2 \% ; \rho_{2}=0.042\right)$,

- $N_{0}=0.704$. This value is the proportion of the normal weight population of 23 years old in the region of Valencia in year 2000 [2].

- $S_{0}=0.25$. This value is the proportion of the overweight population of 23 years old in the region of Valencia in year 2000 [2].

- $O_{0}=0.046$. This value is the proportion of the obese population of 23 years old in the region of Valencia in year 2000 [2].

Additionally, taking as the initial condition of the model (year 2000 -last week-, i.e., $t=0), N(t=0)=0.522, S(t=0)=0.362$ and $O(t=0)=0.116$ and the final condition of the model (year 2005 -last week-, i.e., $t=260$ ), $N(t=260)=0.485, S(t=260)=0.378$ and $O(t=260)=0.137$ the parameters $\beta$ and $\gamma$ have been estimated by fitting the model and we obtained $\beta=0.001121$ and $\gamma=0.0003226$. It is noteworthy to mention that the value $1 / \gamma=3099.59$ weeks $=59.6074$ years, means that an average overweight individual needs 59.6 years to become an obese individual. In Table 1 we summarise the estimated parameters.

Table 1: Model parameters

\begin{tabular}{cc}
\hline Parameter & Value \\
\hline$\mu$ & 0.0004578 \\
$\gamma$ & 0.0003226 \\
$\epsilon_{1}$ & 0.004 \\
$\epsilon_{2}$ & 0.024 \\
$\rho_{1}$ & 0.020 \\
$\rho_{2}$ & 0.042 \\
$p$ & $1 / 7$ \\
$\beta$ & 0.001121 \\
$N_{0}$ & 0.704 \\
$S_{0}$ & 0.25 \\
$O_{0}$ & 0.046 \\
\hline
\end{tabular}




\section{Results}

\subsection{Next few years prediction}

Now, once the model is stated and the parameters estimated, we are able to give predictions of each of the subpopulations over the next few years computing the solutions of the model, $N(t), S(t)$ and $O(t)$ for values of time $t$ in the future. If there are not changes in the population nutritional habits and the public health strategies to reduce weight over the next years, the fraction of normal weight, overweight and obese people in the Spanish region of Valencia will be the ones given in Table 2. Note that normal weight subpopulation is decreasing over time whereas overweight and, overall, obese subpopulations are increasing.

Table 2: Predictions of normal weight, overweight and obese people in the Spanish region of Valencia at the end of next years 2013, 2015 and 2030. Note the increasing proportion of subpopulations $S$ and $O$.

\begin{tabular}{cccc}
\hline Subpopulation & 2013 & 2015 & 2030 \\
\hline$N$ & 0.4350 & 0.4244 & 0.3657 \\
$S$ & 0.3970 & 0.4004 & 0.4132 \\
$O$ & 0.1679 & 0.1751 & 0.2210 \\
\hline
\end{tabular}

\subsection{Random prediction over the next few years}

Now, let us calculate predictions introducing randomness in the parameters and the initial conditions of the model. The introduction of randomness in the aforementioned model (1)-(3) is justified by the fact that parameters and initial conditions have some degree of uncertainty (sampling and rounding errors) and, consequently, the predictions are involved with the uncertainty. Thus, the predictions are usually given by confidence intervals.

To do that, typically, it is more appropriate to express the parameter values in terms of probability distributions. When few data are available, like our case, it is usually admitted the use of the uniform probability distribution centred in the corresponding estimated values of Table 1 . Table 3 shows details about probability distribution of the input parameters. Variation range for $\beta$ and $\gamma$ are defined assuming an estimation error not greater than $20 \%$. The variation range of the other parameters is defined by binomial proportion confidence intervals [16]. In particular, to determine the variation intervals for the initial conditions, data from the Health Survey of the region of Valencia (year 2000) [2] have been considered.

In order to compute random predictions, to be precise $90 \%$ confidence intervals (90\% CI), we use the technique called Latin Hypercube Sampling (LHS) $[17,18]$. Latin Hypercube Sampling (a type of stratified Monte Carlo sampling) is a sophisticated and efficient method for achieving equitable sample of all input parameters simultaneously. Each input parameter (transmission parameters 
Table 3: Parameter distributions. We assume uniform probability distribution centred in the deterministic estimated values. Variation range for $\beta$ and $\gamma$ are defined assuming an estimation error not greater than $20 \%$. The variation range of the other parameters is defined by binomial proportion confidence intervals [16].

\begin{tabular}{cccc}
\hline Parameter & Deterministic value & Interval & Distribution \\
\hline$\mu$ & 0.000469 & - & - \\
$\beta$ & 0.001121 & {$[0.0008970,0.001345]$} & Uniform \\
$\gamma$ & 0.0003226 & {$[0.0002581,0.0003871]$} & Uniform \\
$\epsilon_{1}$ & 0.004 & {$[0.0021,0.0058]$} & Uniform \\
$\epsilon_{2}$ & 0.024 & {$[0.019,0.028]$} & Uniform \\
$\rho_{1}$ & 0.020 & {$[0.015,0.024]$} & Uniform \\
$\rho_{2}$ & 0.042 & {$[0.036,0.047]$} & Uniform \\
$p$ & $1 / 7$ & {$[1 / 9,1 / 5]$} & Uniform \\
$N_{0}$ & 0.70 & {$[0.69,0.71]$} & Uniform \\
$S_{0}$ & 0.25 & {$[0.23,0.26]$} & Uniform \\
$O_{0}$ & 0.046 & {$[0.039,0.052]$} & Uniform \\
$N(t=0)$ & 0.522 & {$[0.507,0.536]$} & Uniform \\
$S(t=0)$ & 0.362 & {$[0.347,0.376]$} & Uniform \\
$O(t=0)$ & 0.116 & {$[0.106,0.125]$} & Uniform \\
\hline
\end{tabular}

and initial conditions) is defined as having a probability density function associated with it. Then, the model can be simulated by sampling a single value from each parameter distribution. Many samples should be taken and many simulations should be run producing variable output values. In our case, LHS was used to generate 5,000 different values of each input parameter. We used these samples to run 5,000 evaluations of the deterministic model. The results of these evaluations allow us to determine output $90 \%$ confidence intervals, obtained taking into account the empirical $5 \%$ and $95 \%$ percentiles. The obtained $90 \%$ confidence interval predictions appear in Table 4 . Note two facts: Table 4 shows similar trends to the ones observed in Table 2; longer term predictions give larger confident intervals.

Table 4: 90\% confidence interval predictions for normal weight, overweight and obese people in the Spanish region of Valencia for next years 2013, 2015 and 2030. Analogously to Table 2 , there is an increasing proportion of subpopulations $S$ and $O$.

\begin{tabular}{cccc}
\hline Subpopulation & 2013 & 2015 & 2030 \\
\hline$N$ & {$[0.4055,0.4663]$} & {$[0.3924,0.4590]$} & {$[0.3217,0.4168]$} \\
$S$ & {$[0.3625,0.4254]$} & {$[0.3632,0.4309]$} & {$[0.3633,0.4517]$} \\
$O$ & {$[0.1525,0.1817]$} & {$[0.1583,0.1899]$} & {$[0.1942,0.2442]$} \\
\hline
\end{tabular}




\subsection{Health communication campaigns to be simulated}

Now, let us define several possible policies to be developed by the Valencian Health Authorities and implemented by means of health communication campaigns in order to convince the population to change to healthier lifestyles and, therefore, to reduce weight. Each one of these policies is going to be simulated and the effect on the subpopulations estimated and compared.

Then, taking into account that excess weight control policies can be described by the parameters of the model, let us perturb the value of the parameters in the right way to simulate the application of each health campaign to determine the effects of such changes over the next few years.

Here, it is important to recall that a health campaign change the behaviour of about $5 \%$ of the population in the expected direction $[19,20,21]$. Nevertheless, the referred changes are related to population, not to parameters and therefore, not concerned to transition rates, except for parameters $\rho_{1}, \rho_{2}, \epsilon_{1}, \epsilon_{2}$, (percentage of overweight and obese people who are on diet or doing exercise) $N_{0}, S_{0}, O_{0}$ (new recruited 24-year-old individuals) and the initial conditions. However, as we mentioned before, policies and strategies are designed to change the value of the model parameters. Thus, even though we do not have any real quantification of the other parameters $(\beta, \gamma$ and $p)$, let us assume that all parameters corresponding to a certain policy may have a variation not greater than $5 \%$ of their values, i.e., we consider a variation of $5 \%$ of the intervals given in Table 3 in the appropriated direction.

In order to reduce the excess weight prevalence, the following obesity control proposals are simulated:

1. Promotion to maintain healthy lifestyles in normal weight subpopulation. This is a preventive policy that seeks to reduce the flow of normal weight individuals to overweight, which implies a reduction of $5 \%$ in the interval of parameter $\beta$, i.e., $\beta$ moves uniformly in the interval [0.0008521, 0.0012782].

2. Promotion to maintain lifestyles in normal weight and overweight subpopulations. This is a combination of preventive campaigns seeking to reduce the transit from normal and overweight to overweight and obese, respectively, which implies a reduction of $5 \%$ in the interval of parameters $\beta$ and $\gamma$. Thus, $\beta$ moves uniformly in the interval $[0.0008521,0.0012782]$ and $\gamma$ inside [0.0002452, 0.0003678].

3. Promotion to maintain healthy lifestyles in normal weight and to encourage overweight subpopulation to do exercise and put on diet. This is a mixture of preventive and treatment campaigns looking for reducing the transit from normal weight to overweight and increasing the return flow from overweight to normal weight subpopulation, involving a $5 \%$ decrease of the parameter $\beta$ and a $5 \%$ increase of $\rho_{1}$ and $\rho_{2}$, moving uniformly in the intervals [0.0008521, 0.0012782], [0.01575, 0.0252] and [0.0378, 0.04935], respectively.

4. Promotion to maintain healthy lifestyles in normal weight and to encourage overweight and obese subpopulations to do exercise and put on diet. This is also a mixture of preventive and treatment campaigns in order to reduce 
the transit from normal weight to overweight and increasing the return flow from overweight and obese subpopulations. This campaign involves a $5 \%$ decrease of the parameter $\beta$ and a $5 \%$ increase of parameters $\rho_{1}, \rho_{2}, \epsilon_{1}$ and $\epsilon_{2}$, all together, moving uniformly in the intervals [0.00085, 0.00127], [0.01575, 0.0252], [0.002205, 0.00609], [0.0378, 0.04935] and [0.01995, 0.0294], respectively.

5. Promotion to put overweight and obese individuals on diet. This is a treatment campaign to increase the number of individuals who transit from obese or overweight to overweight or normal weight, respectively. It involves a $5 \%$ increase of parameters $\rho_{2}$ and $\epsilon_{2}$ all together, varying uniformly inside the intervals [0.002205, 0.00609] and [0.01995,0.0294], respectively.

6. Promotion of physical activity in overweight and obese subpopulation. This is also a treatment campaign. Its objective is to increase the number of individuals who transit from obese or overweight to overweight or normal weight, respectively. It involves a $5 \%$ increase of parameters $\rho_{1}$ and $\epsilon_{1}$ all together, varying uniformly in the intervals $[0.01575,0.0252]$ and [0.0378, 0.04935], respectively.

7. Promoting overweight and obese individuals to do exercise and put on diet. This campaign is the addition of the two previous. Then, it is associated with the parameters $\rho_{2}, \epsilon_{2}, \rho_{1}$ and $\epsilon_{1}$ all together. To vary this parameters we assume that they follow a uniform probability distribution with support on the intervals $[0.01575,0.0252],[0.002205,0.00609],[0.0378,0.04935]$ and [0.01995, 0.0294], respectively.

\section{Discussion}

Once the health communication campaigns have been defined and quantified, we apply the LHS technique to 5,000 samples using uniform distributions on the intervals that determine each campaign and compute, with the obtained 5,000 outputs, the $90 \%$ condence interval, the mean and the median. In order to make a comparison between the obtained results of all the policies and strategies considered and taking into account that the confidence intervals obtained are of different lengths, let us compare the medians. The results can be seen in Table 5 .

The best result are given by health campaigns 3 and 4 (increase of normal weight subpopulation of: $0.97 \%$ and $0.99 \%$ in $2013 ; 1.08 \%$ and $1.10 \%$ in 2015 ; $1.64 \%$ and $1.62 \%$ in 2030), because in both there are a larger increase in normal weight and decrease of excess weight subpopulations. This is reasonable because in both policies almost all parameters have been involved in the common goal of weight reduction, combining preventive and treatment strategies.

Following health campaigns 3 and 4, we have 2 and 1, both only preventives, not far from 3 and 4 (increase of normal weight subpopulation of: $0.77 \%$ and $0.74 \%$ in $2013 ; 0.85 \%$ and $0.81 \%$ in $2015 ; 1.29 \%$ and $1.18 \%$ in 2030 ). Campaign 2 is better than 1 because involves more parameters.

Finally, the treatment campaigns 7,5 and 6, whose effect are significantly less than the preventive ones 2 and 1 (increase of normal weight subpopulation of: 
Table 5: Table with the proposed health communication campaigns against subpopulations. Difference between medians obtained applying the health communication campaigns and medians without applying these policies, at the end of the years 2013, 2015 and 2030. These differences correspond to percentages of the total population.

\begin{tabular}{|c|c|c|c|c|c|c|c|}
\hline \multicolumn{8}{|c|}{2013} \\
\hline & 1 & 2 & 3 & 4 & 5 & 6 & 7 \\
\hline$N$ & $0.74 \%$ & $0.77 \%$ & $0.97 \%$ & $0.99 \%$ & $0.12 \%$ & $0.07 \%$ & $0.22 \%$ \\
\hline$S$ & $-0.64 \%$ & $-0.35 \%$ & $-0.92 \%$ & $-0.89 \%$ & $-0.08 \%$ & $-0.07 \%$ & $-0.22 \%$ \\
\hline$O$ & $-0.07 \%$ & $-0.38 \%$ & $-0.09 \%$ & $-0.11 \%$ & $-0.02 \%$ & $-0.04 \%$ & $-0.04 \%$ \\
\hline \multicolumn{8}{|c|}{2015} \\
\hline & 1 & 2 & 3 & 4 & 5 & 6 & 7 \\
\hline$N$ & $0.81 \%$ & $0.85 \%$ & $1.08 \%$ & $1.10 \%$ & $0.10 \%$ & $0.08 \%$ & $0.23 \%$ \\
\hline$S$ & $-0.71 \%$ & $-0.39 \%$ & $-0.99 \%$ & $-0.99 \%$ & $-0.08 \%$ & $-0.07 \%$ & $-0.23 \%$ \\
\hline$O$ & $-0.09 \%$ & $-0.44 \%$ & $-0.12 \%$ & $-0.15 \%$ & $-0.04 \%$ & $-0.06 \%$ & $-0.04 \%$ \\
\hline \multicolumn{8}{|c|}{2030} \\
\hline & 1 & 2 & 3 & 4 & 5 & 6 & 7 \\
\hline$N$ & $1.18 \%$ & $1.29 \%$ & $1.64 \%$ & $1.62 \%$ & $0.18 \%$ & $0.17 \%$ & $0.45 \%$ \\
\hline$S$ & $-0.92 \%$ & $-0.42 \%$ & $-1.29 \%$ & $-1.32 \%$ & $-0.13 \%$ & $-0.14 \%$ & $-0.30 \%$ \\
\hline$O$ & $-0.27 \%$ & $-0.82 \%$ & $-0.36 \%$ & $-0.39 \%$ & $-0.08 \%$ & $-0.10 \%$ & $-0.15 \%$ \\
\hline
\end{tabular}

$0.22 \%, 0.12 \%$ and $0.07 \%$ in $2013 ; 0.23 \%, 0.10 \%$ and $0.08 \%$ in $2015 ; 0.45 \%, 0.18 \%$ and $0.17 \%$ in 2030). Among the treatment campaigns, the 7 is the best because involves all the treatment parameters. Between 5 and 6,5 is better, which indicates that health campaigns focusing on diet for excess weight individuals are more effective than those focusing on the promotion of physical activities. It may be explained because the Health Survey indicates that there are more excess weight people on diet than doing exercise regularly. However, the health campaign 7 which combine both aspects (diet + physical activity) has better repercussion (increase of normal weight subpopulation of $0.23 \%$ in 2015) than the sum of both (increase of normal weight subpopulation of campaign $5+$ campaign $6=0.10 \%+0.08 \%=0.18 \%$ in 2015). This also occurs in health campaigns 1,3 and 7 , where there are more people who reduce weight under the campaign 3 (increase of normal weight subpopulation of $1.08 \%$ in 2015) than the people under the combined effects of 1 and 7 separately (increase of normal weight subpopulation of campaign $1+$ campaign $7=0.81 \%+0.23 \%=1.04 \%$ in 2015).

\section{Conclusion}

In this article, we study the evolution of the proportion of adult valencian inhabitants (24-65 years old) with excess weight over the next few years and examine the impact of some obesity control strategies in reducing excess weight 
subpopulation. The analysed control strategies are based on health communication campaigns following the proposals considered by the Valencian Department of Health in the Valencian Health Plan (2005-2009) [5].

To do that, we propose an epidemiological mathematical model to study the obesity evolution in the region of Valencia (Spain). This model considers excess weight as a communicable disease that spreads by social transmission of unhealthy lifestyles. Valencian population are divided into supopulations of interest where certain parameters determine the transitions between these subpopulations. Furthermore, the parameters are associated with the seven health policies considered to be simulated.

Three main conclusions have been established:

- The transitions from normal weight to overweight and obese are slow, but the paths to get normal weight again are slower, therefore, preventive health campaigns in order to avoid people increasing their intake are more effective than treatment ones.

- Combination of preventive plus treatment campaigns are more effective than considering them separately.

- The simulations lead us to say that health campaigns focusing on diet are more effective than those focusing on the promotion of physical activities. It may happen because data from the Health Survey indicates that are more people on diet than practising exercise regularly. This suggests that the Public Health Authorities should promotion seriously the physical activity (as a preventive and treatment health policy) among population.

Our next step is to propose an age and gender structured model to study more precisely the effect of the health communication campaigns on each subpopulation.

Finally, this paper is an example about how models can be an useful tool to experiment with health policy proposals. Using this type of approach, health policy makers are able to simulate different situations and analyse the effect of changes.

\section{Acknowledgements}

The authors acknowledge the collaboration of Valencian Department of Health and Valencian Economic Research Institute (IVIE). This work has been supported by 2010 Contract for Research Program of Valencian Economic Research Institute (IVIE).

\section{References}

[1] WHO (2010). World Health Organization. Global strategy on diet, physical activity and health [Technical report]. Available at http://www.who.int/dietphysicalactivity/publications/facts/obesity/en (Accessed 16 March 2010). 
[2] Valencian Department of Health. Health Survey, year 2000 (2000). Available at http://www.san.gva.es/val/prof/homeprof.html (Accessed 16 March 2010).

[3] Valencian Department of Health. Health Survey, year 2005 (2005a). Available at http://www.san.gva.es/val/prof/homeprof.html (Accessed 16 March 2010).

[4] J. Aranceta, L. Serra, M. Foz, B. Moreno, Prevalencia de la obesidad en España [Obesity prevalence in Spain]. Medicina Clínica 125 (2005) 460-466.

[5] Valencian Department of Health. Health Plan 2005-2009 (2005b). Available at http://www.san.gva.es/cas/comun/plansalud/indice.htm (Accessed 16 March 2010).

[6] N.A. Christakis, J.H. Fowler, Connected: the surprising power of our social networks and how they shape our lives, Brown and Company. Hachette Book Group, 2009.

[7] N.A. Christakis, J.H. Fowler, The spread of obesity in a large social network over 32 years, The New England Journal of Medicine, 357 (2007) 370-379.

[8] A.M. Evangelista, A.R. Ortiz, K.R. Rios-Soto, A. Urdapilleta. U.S.A. the fast food nation: obesity as an epidemic. http://math.lanl.gov/SummerPrograms/Reports2004/ev_or_ri_ur.pdf, 2004 .

[9] L. Jodar, F.J. Santonja, G. Gonzalez. Modeling dynamics of infant obesity in the region of Valencia, Spain. Computers and Mathematics with Aplications, 56:679 -689, 2008.

[10] G. Gonzalez, L. Jodar, F.J. Santonja, R.J. Villanueva. Age-structured model for childhood obesity. Mathematical Population Studies, 17:1 11, 2010 .

[11] F.J. Santonja, R.J. Villanueva, L. Jódar, G. González, Mathematical modeling of social obesity epidemic in the region of Valencia, Spain, Mathematical and Computer Modelling of Dynamical Systems, 16 (2010) 23 34 .

[12] E. Sánchez, R.J. Villanueva, F.J. Santonja, M. Rubio, Predicting cocaine consumption in Spain. A mathematical modeling approach, Drugs: Education, Prevention \& Policy (Accepted. To be published).

[13] J.D. Murray, Mathematical Biology, Springer, 2002.

[14] M.A. Rubio, J. Salas, M. Barbany, et al., Consenso SEEDO 2007 para la evaluació del sobrepeso y la obesidad y el establecimiento de criterios de intervención terapeútica (Consensus to evaluate overweight and obesity in Spain, year 2007), Revista Española de Obesidad, 3 (2007) 7-47. 
[15] W.C. Miller, D.M. Koceja, E.J. Hamilton, A meta-analysis of the past 25 years of weight loss research using diet, exercise or diet plus exercise intervention, International Journal of Obesity, 21 (1997) 941-947.

[16] R.V. Hogg, J.W. McKean, A.T. Craig, Introduction to Mathematical Statistics, Prentice Hall, 2005.

[17] A. Hoare, D.G. Regan, D.P. Wilson, Sampling and sensitivity analyses tools (SaSAT) for computational modelling, Theoretical Biology and Medical Modelling, 5 (2008) 1-18.

[18] S. Marino, I.B. Hogue, C. J. Ray, D.E. Kirschner, A methodology for performing global uncertainty and sensitivity analysis in systems biology, Journal of Theoretical Biology, 254 (2008) 178-196.

[19] A. Bauman, B.J. Smith, E.W. Maibach, B. Reger-Nash, Evaluation of mass media campaings for physical activity, Evaluation and Program Planning, 29 (2006) 312-322.

[20] S.M. Noar, A 10-year retrospective of research in health mass media campaings: where do we go from here?, Journal of Helath Communication, 11 (2006) 21-42.

[21] L.B. Snyder, Health comunication campaings and their impact on behavior, Journal of Nutrition Education and Behavior, 39 (2007) 32-40. 